\title{
Prostate Cancer Molecular Imaging Standardized Evaluation (PROMISE): Proposed miTNM Classification for the Interpretation of PSMA-Ligand PET/CT
}

\author{
Matthias Eiber ${ }^{1,2}$, Ken Herrmann ${ }^{1,3}$, Jeremie Calais ${ }^{1}$, Boris Hadaschik ${ }^{4}$, Frederik L. Giesel ${ }^{5}$, Markus Hartenbach ${ }^{6}$, \\ Thomas Hope ${ }^{7}$, Robert Reiter ${ }^{8}$, Tobias Maurer ${ }^{9}$, Wolfgang A. Weber ${ }^{10}$, and Wolfgang P. Fendler ${ }^{1,11}$ \\ ${ }^{1}$ Department of Molecular and Medical Pharmacology, David Geffen School of Medicine at UCLA, Los Angeles, California; \\ ${ }^{2}$ Department of Nuclear Medicine, Klinikum Rechts der Isar, Technical University of Munich, Munich, Germany; ${ }^{3}$ Klinik für \\ Nuklearmedizin, Universitätsklinikum Essen, Essen, Germany; ${ }^{4}$ Department of Urology, Universitätsklinikum Essen, Essen, \\ Germany; ${ }^{5}$ Department of Nuclear Medicine, University Hospital Heidelberg and DKFZ Heidelberg, Heidelberg, Germany; ${ }^{6}$ Division \\ of Nuclear Medicine, Department of Biomedical Imaging and Image-Guided Therapy, Medical University of Vienna, Vienna, Austria; \\ ${ }^{7}$ Department of Radiology and Biomedical Imaging, University of California, San Francisco, San Francisco, California; ${ }^{8}$ Department \\ of Urology, David Geffen School of Medicine at UCLA, Los Angeles, California; ${ }^{9}$ Department of Urology, Klinikum Rechts der Isar, \\ Technical University of Munich, Munich, Germany; ${ }^{10}$ Molecular Imaging and Therapy Service, Department of Radiology, Memorial \\ Sloan Kettering Cancer Center, New York, New York; and ${ }^{11}$ Department of Nuclear Medicine, Ludwig-Maximilians-University of \\ Munich, Munich, Germany
}

See an invited perspective on this article on page 466.

Prostate-specific membrane antigen (PSMA)-ligand PET imaging provides unprecedented accuracy for whole-body staging of prostate cancer. As PSMA-ligand PET/CT is increasingly adopted in clinical trials and routine practice worldwide, a unified language for image reporting is urgently needed. We propose a molecular imaging TNM system (miTNM, version 1.0) as a standardized reporting framework for PSMA-ligand PET/CT or PET/MRI. miTNM is designed to organize findings in comprehensible categories to promote the exchange of information among physicians and institutions. Additionally, flowcharts integrating findings of PSMA-ligand PET and morphologic imaging have been designed to guide image interpretation. Specific applications, such as assessment of prognosis or impact on management, should be evaluated in future trials. miTNM is a living framework that evolves with clinical experience and scientific data.

Key Words: PROMISE; miTNM; PSMA-ligand PET/CT; standardized evaluation; interpretation; criteria

J Nucl Med 2018; 59:469-478

DOI: 10.2967/jnumed.117.198119

\section{$\mathbf{P}$} rostate-specific membrane antigen (PSMA)-ligand PET/CT or PET/MRI provides high sensitivity and specificity for prostate cancer staging (1). The accuracy of PSMA-ligand hybrid imaging is superior to that of conventional imaging and tracers such as choline across a range of indications and disease extents (2-15).

Received Jun. 23, 2017; revision accepted Oct. 5, 2017.

For correspondence or reprints contact: Wolfgang Peter Fendler, University of California at Los Angeles, Ahmanson Translational Imaging Division, 10833

Le Conte Ave., 200 Medical Plaza, Los Angeles, CA 90095.

E-mail: wfendler@mednet.ucla.edu

Guest Editor: David Mankoff, University of Pennsylvania.

Published online Nov. 9, 2017.

COPYRIGHT (C) 2018 by the Society of Nuclear Medicine and Molecular Imaging.
Level $2 \mathrm{~b}$ evidence for superior detection rates at early biochemical recurrence after radical prostatectomy led to a grade A recommendation for PSMA-ligand PET/CT by the European Association of Urology (16). We anticipate increased adoption of PSMA-ligand PET/CT fueled by upcoming evidence and inclusion into guidelines. Thus, reporting standards must be created now to aid reproducibility, enhance communication, and ultimately support acceptance of this technology to the benefit of prostate cancer patients.

The Prostate Cancer Molecular Imaging Standardized Evaluation (PROMISE) criteria reported in this issue of The Journal of Nuclear Medicine summarize standards for study design and reporting of prostate cancer molecular imaging. We acknowledge that performance characteristics from different studies can be compared only if target regions are properly described and uniformly used. Therefore, PROMISE recommends that definition of anatomic regions be guided by reproducibility, general applicability, and clinical relevance. Uniform frameworks for image reporting have previously been proposed for pelvic multiparametric MRI (17), bone scintigraphy (18), and many other techniques and indications $(19,20)$. Precise description and organized classification of PSMA-ligand PET/CT findings are needed to serve both clinical reporting (to help with defining tumor extent, tailoring therapy, assessing prognosis, and facilitating exchange of information between centers) and research (to help with validating findings, pooling data within multicenter trials, and performing metaanalyses of published data).

The clinicopathologic TNM system of the American Joint Committee on Cancer and Union Internationale Contre le Cancer is the most widely used prostate cancer staging system (21). In clinical practice, the TNM score is based on a patchwork of information: local, nodal, and distant involvement are categorized by histopathologic examination after surgery or other tissue sampling, as well as clinical findings and imaging. Combination of all modalities improves staging, as each single modality comes with limitations: in prostate cancer clinical examination, ultrasound, CT, and MRI have a low sensitivity for metastases (22), whereas surgery and biopsy with subsequent histopathologic examination 
TABLE 1

miPSMA Expression Score

\begin{tabular}{|c|c|c|}
\hline Score & $\begin{array}{l}\text { Reported PSMA } \\
\text { expression }\end{array}$ & Uptake \\
\hline 0 & No & Below blood pool \\
\hline 1 & Low & $\begin{array}{l}\text { Equal to or above blood } \\
\text { pool and lower than liver* }\end{array}$ \\
\hline 2 & Intermediate & $\begin{array}{l}\text { Equal to or above liver }{ }^{*} \text { and } \\
\text { lower than parotid gland }\end{array}$ \\
\hline 3 & High & $\begin{array}{l}\text { Equal to or above parotid } \\
\text { gland }\end{array}$ \\
\hline
\end{tabular}

${ }^{*}$ For PSMA ligands with liver-dominant excretion (e.g., ${ }^{18} \mathrm{~F}-$ PSMA1007) spleen is recommended as reference organ instead of liver.

can evaluate only the dissected tissue and, thus, often underdiagnose prostate cancer metastases at extrapelvic regions or locations outside the operating or sampling field (23).

Detection of prostate cancer with PSMA-ligand PET/CT depends on target expression. On the basis of the high and specific target expression level of most prostate cancer cells, PSMA-ligand PET/CT detects more than $50 \%$ of lymph node metastases with a short diameter of at least $2.3 \mathrm{~mm}$ and more than $90 \%$ of those with a short diameter of at least $4.5 \mathrm{~mm}$ in a salvage lymphadenectomy setting (24). Staging is provided for the entire field of view and for regions otherwise inaccessible by surgery or biopsy. In view of these unique characteristics, we propose a molecular imaging TNM (miTNM) framework for PSMA-ligand PET/CT prostate cancer staging. This framework may also be applied for PSMAligand PET/MRI, SPECT/CT, or similar approaches. miTNM serves to provide standardized reporting of the presence, location, and extent of local prostate cancer and its pelvic spread; the presence, location, extent, and distribution pattern of extrapelvic metastases; the PSMA expression level of tumor lesions; and diagnostic confidence about reported findings.

To support acceptance, implementation, and correlation, definitions for the PSMA-ligand PET/CT miTNM framework were designed in analogy with the clinicopathologic TNM framework when possible. Categories describing the PSMA expression level and pattern of bone involvement were added-for example, to aid the planning of PSMA-directed therapy or the estimation of patient prognosis.

PSMA-ligand PET/CT provides high accuracy at substantial to almost-perfect reproducibility for TNM staging among readers with various levels of experience (25). Precise and reproducible staging was achieved even without detailed criteria for lesion positivity (25). Nevertheless, for successful application of miTNM in prostate cancer staging, it is crucial to have criteria for performing and interpreting PSMA-ligand PET/CT (26-28), as recently

TABLE 2

miTNM Classification for PSMA-Ligand PET/CT or PET/MRI

\begin{tabular}{|c|c|}
\hline Class & Description \\
\hline \multicolumn{2}{|l|}{ Local tumor $(T)$} \\
\hline miT0 & No local tumor \\
\hline miT2 & Organ-confined tumor; report intraprostatic tumor location on sextant basis (Table 3) \\
\hline $\mathrm{u}$ & Unifocality \\
\hline $\mathrm{m}$ & Multifocality \\
\hline miT3 & Non-organ-confined tumor; report intraprostatic tumor location on sextant basis (Table 3 ) \\
\hline a & Extracapsular extension \\
\hline $\mathrm{b}$ & Tumor invading seminal vesicles \\
\hline miT4 & $\begin{array}{l}\text { Tumor invading adjacent structures other than seminal vesicles, such as external sphincter, } \\
\text { rectum, bladder, levator muscles, or pelvic wall }\end{array}$ \\
\hline miTr & Presence of local recurrence after radical prostatectomy \\
\hline \multicolumn{2}{|c|}{ Regional nodes $(\mathrm{N})$} \\
\hline miNO & No positive regional lymph nodes \\
\hline miN1a & $\begin{array}{l}\text { Single lymph node region harboring lymph node metastases; report location by standardized } \\
\text { template (Table 4) }\end{array}$ \\
\hline miN1b & $\begin{array}{l}\text { Multiple }(\geq 2) \text { lymph node regions harboring lymph node metastases; report locations by } \\
\text { standardized template (Table } 4)\end{array}$ \\
\hline \multicolumn{2}{|c|}{ Distant metastases (M) } \\
\hline miM0 & No distant metastasis \\
\hline $\operatorname{miM1}$ & Distant metastasis \\
\hline a & Extrapelvic lymph nodes; additionally report location by standardized miM1a template (Table 4) \\
\hline $\mathrm{b}$ & Bones; additionally report pattern (Table 5) and involved bones if unifocal or oligometastatic \\
\hline $\mathrm{C}$ & Other sites; additionally report involved organ \\
\hline
\end{tabular}


TABLE 3

Sextant Segmentation of Prostate Gland

\begin{tabular}{ll}
\hline Segment & miT2-4 template \\
\hline LB & Left base \\
RB & Right base \\
LM & Left mid \\
RM & Right mid \\
LA & Left apex \\
RA & Right apex \\
\hline
\end{tabular}

recommended in a joint guideline by the European Association of Nuclear Medicine and the Society of Nuclear Medicine and Molecular Imaging (27). Therefore, we have designed flowcharts on morphologic and PSMA-ligand PET findings to guide standardized image interpretation.

An overview of miTNM, version 1.0, is given in Tables 1 through 6 and Figures 1 through 4. Anatomic regions and disease patterns are detailed in the following sections.

\section{PSMA EXPRESSION SCORE AND INTERPRETATION CRITERIA}

\section{Rationale}

PSMA expression based on immunohistochemistry is known to correlate with tumor differentiation as well as prognosis (29-31). Loss of PSMA expression in metastases can indicate dedifferentiation and increasing tumor heterogeneity, leading to more aggressive phenotypes and a nonresponse to PSMA-directed therapy $(32,33)$. In intraprostatic lesions, PSMA-ligand PET has been shown to correlate with tumor aggressiveness as defined by the Gleason score $(6,34)$. Absence of PSMA expression as measured by PET in a primary tumor raises concerns about missed PSMA expression in its metastases and therefore provides important information for interpretation of PSMA-ligand PET results $(5,35)$. Thus, information derived from noninvasive mapping of tumoral PSMA expression is valuable and should be reported for clinical and research PSMA-ligand PET.

TABLE 4

Lymph Node Regions

\begin{tabular}{cll}
\hline Region & \multicolumn{1}{c}{ Template } & Report left/right \\
\hline \multicolumn{3}{l}{ miN1a/b } \\
\hline El & Internal iliac & Yes \\
Cl & External iliac & Yes \\
OB & Common iliac & Yes \\
PS & Obturator & Yes \\
OP & Presacral (presciatic) & No \\
& Other pelvic (specify) & No \\
\hline RP & miM1a & \\
\hline SD & Retroperitoneal & No \\
\hline OE & Supradiaphragmatic & Yes or no \\
\hline
\end{tabular}

Details on anatomic definition of lymph node regions are provided in Supplemental Table 1.
TABLE 5

Pattern of Bone Involvement

\begin{tabular}{ll}
\hline Abbreviation & Pattern of bone involvement \\
\hline Uni & Unifocal \\
Oligo & Oligometastatic $(n \leq 3)$ \\
Diss & Disseminated \\
Dmi & Diffuse marrow involvement \\
\hline
\end{tabular}

\section{miPSMA Score}

We propose a miPSMA score that enables standardized reporting of PSMA expression as detected with PSMA-ligand PET. Expression categories are defined in relation to mean uptake in the blood pool, liver, and parotid gland (Table 1; Fig. 1). Results are reported as $0,1,2$, or 3 for no, low, intermediate, or high PSMA expression, respectively. Scores 2 and 3 are empirically considered typical for prostate cancer lesions and favorable for PSMA-directed radioligand therapy. Expression level is determined visually, and we do not recommend uptake measurements on a regular basis. Occasionally, quantitative analyses might be necessary to correctly assign a specific miPSMA score.

On the basis of personal experience, we advise comparison of the mean SUVs of the respective lesions and the reference organ. The liver SUV can be measured by placing a 3-cm-diameter circular region of interest in the normal inferior right liver lobe in the axial plane; the blood pool, by centering a 2-cm-diameter circular region of interest in the aortic arch in the axial plane; the parotid gland, by centering a $1.5-\mathrm{cm}$-diameter circular region of interest in the right parotid gland in the axial plane; and a tumor lesion, by centering a 1-cm-diameter circular region of interest over the voxel with maximum uptake in the axial plane. Notably, SUV measurements in PSMA-ligand PET require further validation and investigation to clarify whether $\mathrm{SUV}_{\text {mean }}, \mathrm{SUV}_{\max }$, or $\mathrm{SUV}_{\text {peak }}$ is the most appropriate parameter.

Detailed comparative data are lacking on the biodistribution of various PSMA ligands. However, application of the miPSMA score for different PSMA ligands appears feasible because their biodistribution is grossly similar (Fig. 1). Known differences in biodistribution (e.g., higher blood-pool activity for ${ }^{18} \mathrm{~F}-\mathrm{DCFBC}$ or higher liver uptake for ${ }^{18}$ F-PSMA1007) should be considered, especially when comparing studies using different ligands. For PSMA

TABLE 6

Certainty and Final Diagnosis

\begin{tabular}{ll}
\hline \multicolumn{1}{c}{ Certainty } & Diagnosis \\
\hline Consistent with & Positive \\
\hline Suggestive of & Positive \\
\hline Possible & Equivocal \\
Unlikely & Negative \\
No evidence of disease & Negative \\
\hline
\end{tabular}

Final diagnosis should be reported as positive or negative for prostate cancer. Equivocal diagnosis should be used only when alternative techniques are available that may reasonably provide clarification. 


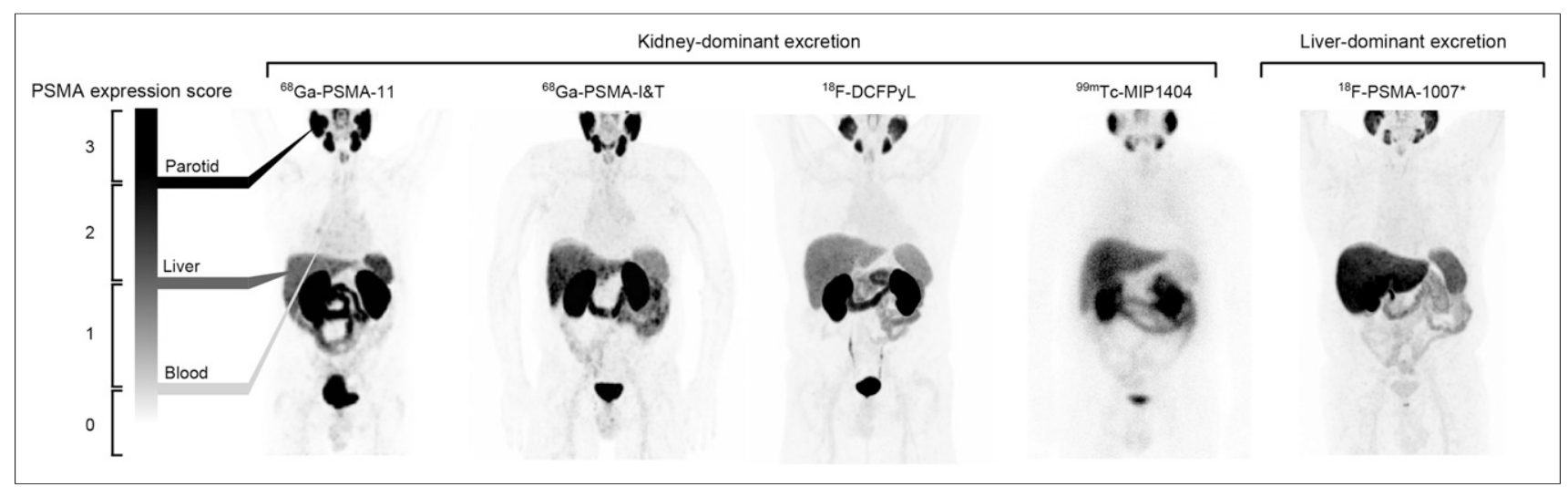

FIGURE 1. miPSMA expression score. Thresholds are demonstrated on ${ }^{68} \mathrm{Ga}-\mathrm{PSMA} 11 \mathrm{PET}$ maximum-intensity projection (left). For comparison,

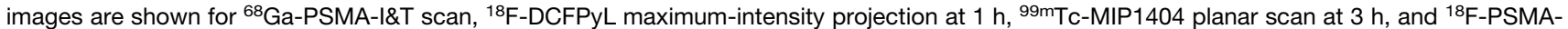
1007 scan. *For PSMA ligands in which the ligand has liver-dominant excretion, spleen is recommended as reference organ instead of liver.

ligands with liver-dominant excretion (e.g., $\left.{ }^{18} \mathrm{~F}-\mathrm{PSMA} 1007\right)$, the spleen is recommended instead of the liver for comparison against blood-pool and salivary gland uptake (36).

\section{Interpretation}

The miPSMA score alone is not suitable for diagnosing or excluding prostate cancer. Interpretation of miPSMA scores must be performed with consideration of the clinical context and other imaging findings and can vary for different tissue classes and even locations. A guide for the interpretation of PSMA-directed imaging based on CT, MRI, and PET findings is given in Figure 2. We designed flowcharts that are based on our clinical experience; however, interpretation critically depends on multiple factors, including indication, current therapy, prostate-specific antigen level, and prior clinical, imaging, or histopathologic findings. The criteria in Figure 2 are not to be taken as absolute definitions for positive, negative, or equivocal findings. Especially in patients with a rising, yet low, level of prostate-specific antigen and otherwise unremarkable imaging findings, even faint but focal uptake above the background level at a typical location may serve as an indicator of prostate cancer. The usability and potential further adoption of the miPSMA score is prone to prospective clinical validation. Definition of more detailed criteria for certain clinical situations, such as was recently proposed using a consensus reading with multiple Delphi rounds (28), is recommended.

The miPSMA score may become useful for selecting patients for targeted radiotherapy. At restaging, a decrease in the miPSMA score in conjunction with morphologic findings can help to identify dedifferentiation or response to therapy.

\section{FINAL DIAGNOSIS AND CERTAINTY}

The final diagnosis should ideally be either positive or negative for prostate cancer. Equivocal findings should be avoided and limited to certain settings, such as when other techniques may be able to clarify the findings. In addition, we recommend reporting diagnostic certainty using a 5-point scale (Table 6). Certainty will substantially vary depending on uptake, location, and CT or MRI findings. For instance, at biochemical recurrence, diagnostic certainty will be substantially higher when focal uptake is at a common location (e.g., internal iliac lymph node) than when at an uncommon location (e.g., rib). Certainty is further influenced by the specific clinical scenario; for example, faint uptake in the prostate gland after radiation therapy may often represent physiologic background activity, whereas any faint uptake in the former prostate bed after radical prostatectomy is highly suggestive.

Standardized wording for the final diagnosis and level of certainty will improve communication between the reader and the treating physician. Implementation into study protocols will allow identification of ambiguous judgments and potential pitfalls, aiding future improvement of PROMISE and miTNM. It will also be desirable to adjust the different categories with data based on studies using histopathologic correlation. This step will increase understanding between corresponding physicians and facilitate any potential consequences, such as a change in management.

\section{LOCAL TUMOR (T)}

Categorization of a local tumor is based on extent and organ confinement (Table 2; Fig. 3A). miT0 describes the absence of local recurrence in the pelvis both after radical prostatectomy and after radiation therapy. miT2 to miT4 categorize tumor extent with the prostate in place, either treated or untreated. Local-organ-confined tumor is defined as miT2 $u$ for unifocal involvement and miT2m for multifocal involvement. Extraprostatic extension is classified by 3 categories in accordance with the clinicopathologic TNM system: limited extraprostatic extension (miT3a), involvement of seminal vesicles (miT3b), and infiltration of external sphincter, rectum, bladder, levator muscles, or pelvic wall (miT4). Because of the low spatial resolution of PET, combination with appropriate crosssectional imaging is needed to adequately judge extraprostatic extension. This is best achieved by complementing PSMA-ligand PET with multiparametric MRI either within a hybrid PET/ MRI study or as a separate dataset available for image fusion. Notably, to avoid confusion with the clinicopathologic TNM system, in which T1 defines a tumor on histopathology with no correlation on palpation or any type of imaging, no miT1 category is used.

To describe the anatomic distribution of intraprostatic tumor extension and to facilitate a straightforward correlation between imaging and histopathology $(6,37)$, information on prostate involvement is described on a sextant basis (Table 3 ). Sextant segments were chosen to provide information for biopsy, the common method of diagnosing prostate cancer. For ultrasound biopsy, 


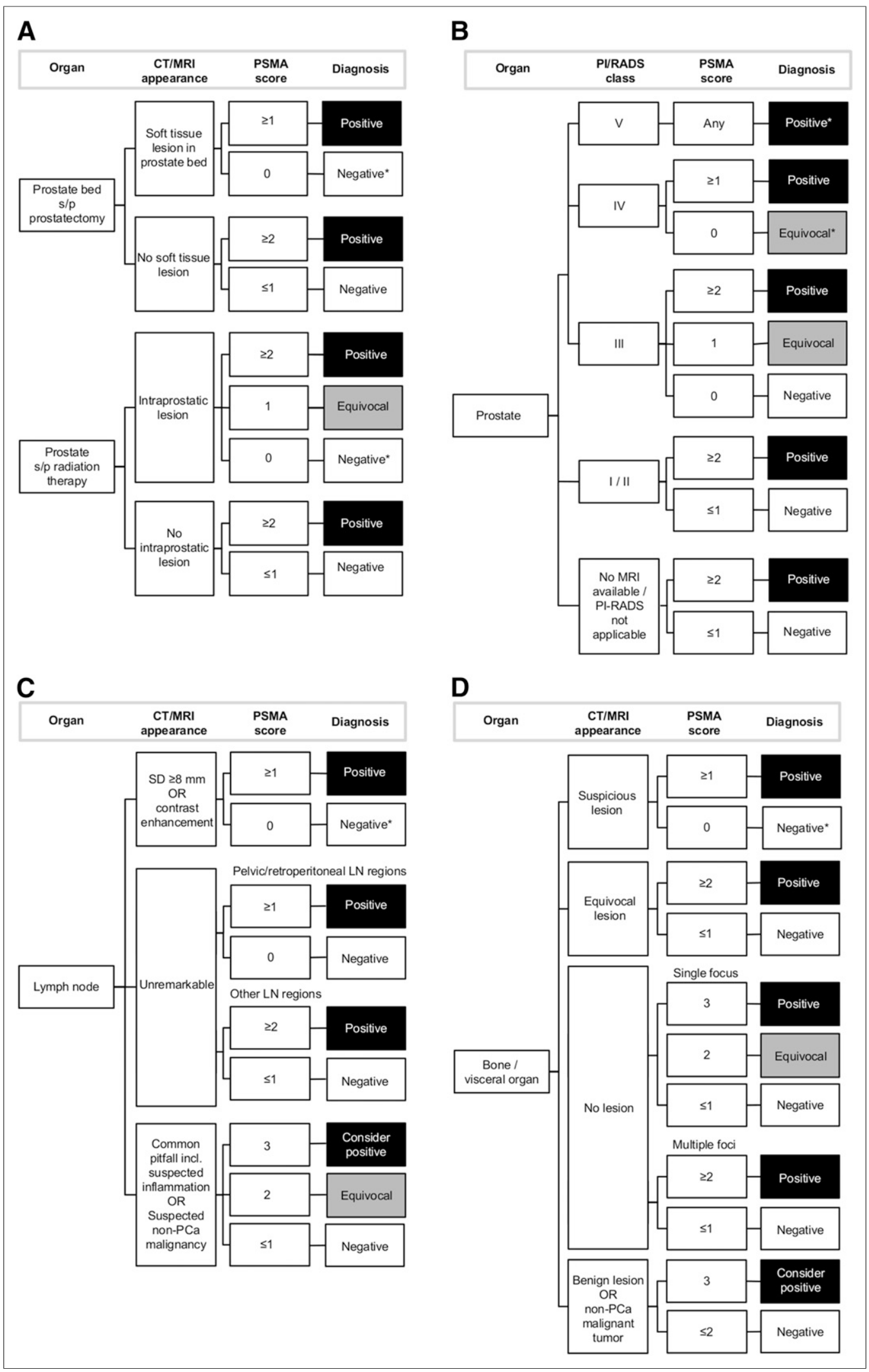

FIGURE 2. Guide for interpretation of PSMA-ligand PET/CT or PET/MRI. Criteria are given separately for imaging of prostate bed after prostatectomy or after radiation therapy $(A)$, imaging of prostate for tumor detection or primary staging of cancer $(B)$, imaging of lymph nodes $(C)$, and imaging of bone or visceral organs $(D)$. $L N=$ lymph node; $P C a=$ prostate cancer; $\mathrm{SD}=$ short-axis diameter; $\mathrm{s} / \mathrm{p}$ = status post. ${ }^{*}$ Consider PSMA-ligand-negative prostate cancer. 


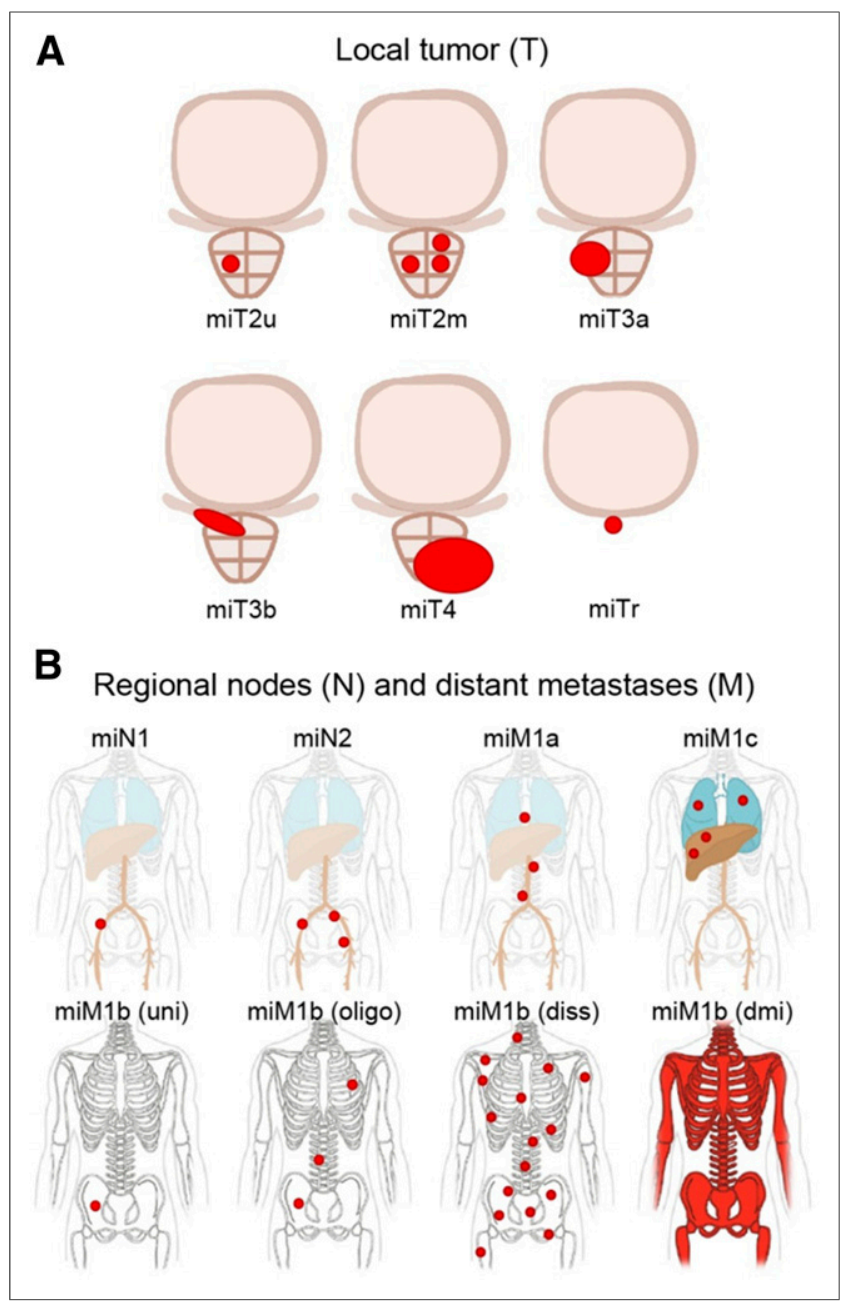

FIGURE 3. miTNM categories and pattern of bone involvement for reporting prostate cancer stage by PSMA-ligand PET/CT: local tumor extent (A) and pelvic node and distant metastases (B). Tumor involvement is delineated in red. Diss = disseminated; $\mathrm{dmi}=$ diffuse marrow involvement; oligo $=$ oligometastatic; uni $=$ unifocal.

image fusion encompassing both cognitive and software-based approaches is recommended (38-41). For traditional sextant segmentation, the craniocaudal extent of the prostate is divided into 3 equal-thickness volumes separated as falling to the left or right of the urethra: that is, left basal, right basal, left mid, right mid, left apical, and right apical segments $(6,42)$. We are aware that more detailed descriptions of intraprostatic tumor involvement exist, such as the local template provided by PI-RADS (the Prostate Imaging and Reporting and Data System, version 2) (17). However, because our system is intended to harmonize image findings across PET/CT and PET/MRI, the sextant approach is most applicable. Outcome data, matched with the pathologic tumor stage, has indicated that tumor extent on a sextant basis or seminal vesicle infiltration is valuable prognostic information $(43,44)$. Nevertheless, in dedicated studies using PET/MRI technology, further discrimination of the prostate gland in the peripheral, transition zone is recommended for reporting of intraprostatic tumor spread, such as by using the proposed template in PI-RADS.

Local recurrence after radical prostatectomy is categorized by miTr. Infiltration of pelvic structures should be detailed in the report. The probability of local tumor both after radical prostatectomy and after radiation therapy increases with focal uptake, higher miPSMA in the prostate (other than the bladder neck or urethra area), MRI showing a typical appearance of local tumor (diffusion restriction, contrast enhancement), or CT showing circumscribed contrast enhancement or signs of extraprostatic extension. A guide integrating the findings of PSMA-ligand PET and morphologic imaging is given in Figure 2A for local tumor after primary treatment and in Figure $2 \mathrm{~B}$ for primary staging or tumor detection. PI-RADS is applicable only for detecting tumor in patients with an increased level of prostate-specific antigen; therefore, it should not be combined with interpretation of PSMA-ligand uptake for primary local staging after histologic confirmation (Fig. 2B). PSMA-ligand-positive pitfalls such as acute prostatitis and MRI-positive pitfalls such as postbiopsy changes and benign nodules must be ruled out. Notably, tumors with a low-Gleason-score pattern, and some rare entities such as intraductal carcinomas, tend to be negative on PSMA-ligand PET.

\section{PELVIC NODES (N)}

Pelvic node metastases are categorized as single involved nodal regions (miN1a) or multiple involved nodal regions (miN1b). Clinical data indicate that the number of metastatic lymph nodes on histopathology significantly affects disease progression and survival (e.g., recurrence-free survival at $10 \mathrm{y}$ of $>70 \%$ vs. $49 \%$ for patients with 1 or 2 vs. $>5$ positive lymph nodes) $(45,46)$. In addition, it is generally accepted that histopathologic information from extended lymph node dissection is important for prognosis (47).

PSMA-ligand PET/CT is currently regarded as the most powerful application for providing a comprehensive overview of nodal involvement in the entire field of view. However, because PSMAligand PET/CT has failed to identify very small $(<2 \mathrm{~mm})$ lymph nodes, we feel that reporting based on traditional surgical templates is appropriate (24). A standardized template for pelvic lymph node regions provides anatomic information to facilitate comparison with surgery, histopathology, or other imaging findings (Table 4; Fig. 4). Such a template covers the different regions usually approached when extended lymph node dissection is performed (23). The anatomic structures delineating template regions for the pelvis, as adopted in two published reports $(48,49)$, are described in Supplemental Table 1 (available at http://jnm. snmjournals.org). Each region is encoded by its initials, with bilateral regions further specified as left or right. Besides having prognostic value for determining disease extent, the specific location of lymph node metastases is critical for surgery and radiation

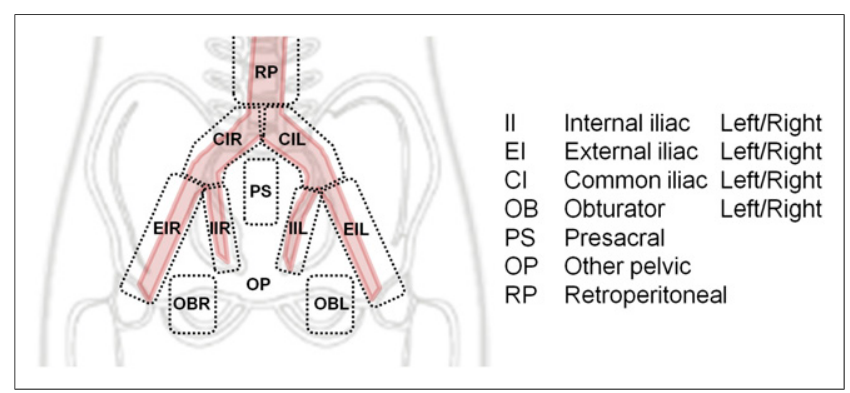

FIGURE 4. miTNM standard template for pelvic lymph node regions. Transition to retroperitoneal extrapelvic region is indicated. 


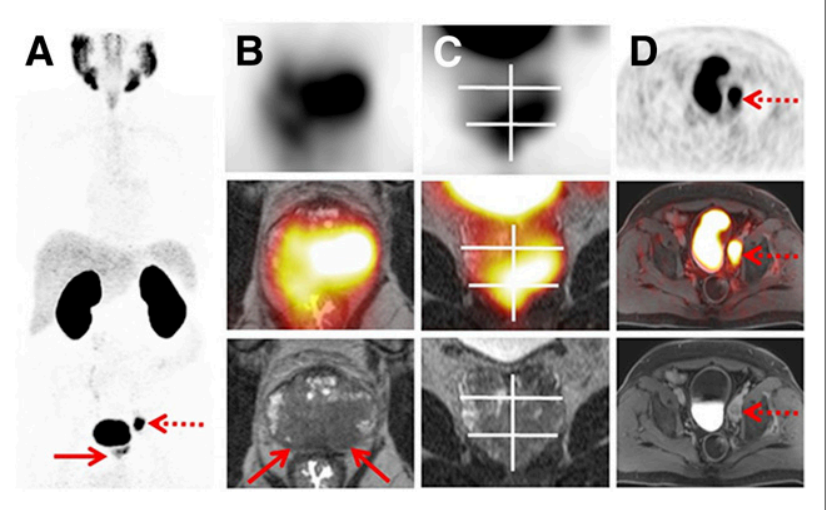

FIGURE 5. Primary staging using ${ }^{68} \mathrm{Ga}-\mathrm{PSMA} 11 \mathrm{PET} /$ high-resolution T2-weighted MRI of prostate in 65-y-old patient with histopathologically proven prostate cancer: PET maximum-intensity projection $(A)$ and axial PET/MRI (B), coronal PET/MRI (C), and axial PET/MRI (PET at top, PET/ MRI in middle, and MRI at bottom). Sextant segment boundaries are shown on coronal images in white. Maximum-intensity projection demonstrates intermediate PSMA expression (score 2) in prostate gland (solid arrow) and high PSMA expression (score 3) in regional pelvic lymph node (dotted arrow). Axial and coronal MRI show bilateral T2hypointense lesions corresponding to uptake on PSMA-ligand PET and clearly exceeding prostate margins, indicative of extraprostatic extension (T3a; solid arrows). Axial and coronal PET/MRI show bilateral involvement of apical and mid segments and of left basal segment on MR images. Axial imaging shows single lymph node metastasis in left obturator region (dotted arrows). Final diagnosis was miT3aN1(OBL)M0. All findings were confirmed by postoperative histopathologic examination.

therapy planning. For instance, the presacral and mesorectal regions, as well as the retroperitoneum, lie outside the typical surgical field.

The probability of nodal involvement increases not only with focal uptake and higher miPSMA score but also with lesion size, contrast enhancement, and location. A guide integrating findings in PSMA-ligand PET and morphologic imaging for pelvic $\mathrm{N}$ staging is given in Figure 2C. CT and MRI abnormalities such as regional grouping, loss of fatty hilum, or focal necrosis may serve as additional morphologic criteria. PSMA-ligand-positive pitfalls such as focal uptake in the celiac ganglia or an adjacent ureter, inflammation, or lymphedema must be ruled out $(7,25,50,51)$.

\section{EXTRAPELVIC NODES AND DISTANT METASTASES (M)}

PSMA-ligand PET/CT detects prostate cancer metastases with better sensitivity and specificity than conventional imaging (1315). At biochemical recurrence, organ involvement can be diagnosed early $(2,8,9)$ and the exact pattern of disease demonstrated. In accordance with the clinicopathologic TNM system, distant metastases are separated into 3 categories: extrapelvic lymph nodes (miM1a), bone metastases (miM1b), and organ metastases (miM1c) (Table 2; Fig. 3B). The location of miM1a nodes is categorized using a standard template (Table 4) as retroperitoneal, supradiaphragmatic, or other. Other lymph node regions or all affected organs in patients with organ involvement (miM1c) should be further specified in the final report.

PSMA-ligand PET/CT has been shown to be superior to bone scintigraphy in describing the extent of bone involvement (13). Bone disease is subcategorized as showing unifocal involvement, oligometastatic involvement, disseminated involvement, or diffuse marrow involvement (Table 5; Fig. 3B). Oligometastatic bone involvement is diagnosed when there are 3 or fewer bone lesions (52). When involvement is unifocal or oligometastatic, the involved bones should be specified. We acknowledge that the concept and final definition of oligometastatic disease are still under debate and that, for example, certain authors count all types of metastatic lesions up to a specific threshold (53). The pattern of bone involvement can have important implications for prognosis $(52,54)$ and management $(53)$. For instance, unifocal involvement may be targetable with curative intent by external-beam radiation therapy, and diffuse marrow involvement indicates elevated risk for hematotoxicity after radionuclide therapy (55-57).

The probability of bone or organ involvement increases with focal uptake, higher miPSMA score, and abnormalities on CT or MRI. For bone metastases, common CT findings include sclerotic, rarely lytic lesions with or without extraosseous extension, and common MRI findings include a low signal on unenhanced T1weighted images. A guide integrating findings on PSMA-ligand PET and morphologic imaging for M-staging is given in Figure 2D. PSMA-ligand-positive pitfalls such as posttraumatic rib uptake and primary malignancies not related to prostate cancer must be ruled out (26). A comprehensive overview of the potential pitfalls for PSMA-ligand PET imaging has recently been published (51).

\section{EXAMPLES}

Figures 5- 7 provide 3 examples illustrating the use of miTNM in different clinical scenarios.

\section{LIMITATIONS}

The aim of miTNM is to create a framework for PSMA-ligand PET reporting. We realize that-like the first clinicopathologic

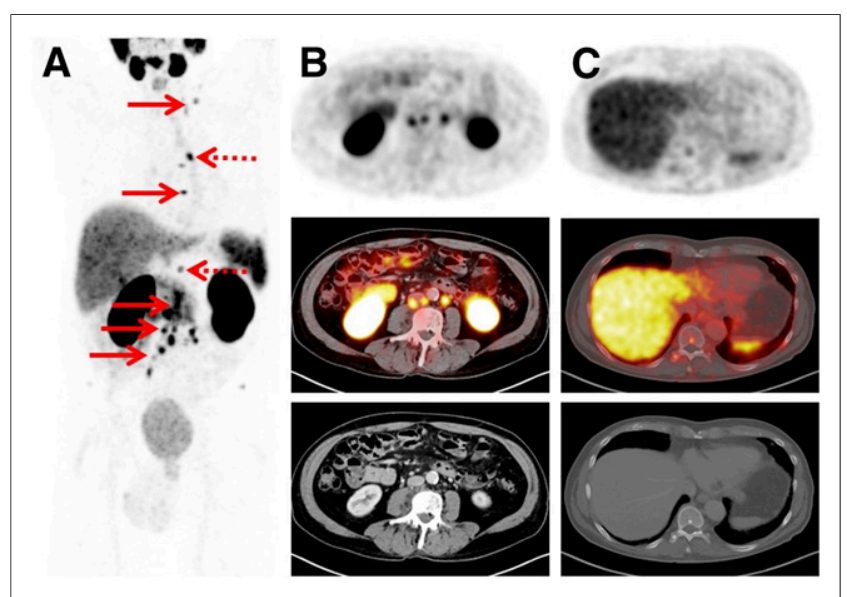

FIGURE 6. ${ }^{68} \mathrm{Ga}-\mathrm{PSMA} 11 \mathrm{PET} / \mathrm{CT}$ restaging in 62-y-old patient with biochemically recurrent prostate cancer and rising level of prostatespecific antigen: PET maximum-intensity projection (A) and 2 levels of axial PET/CT (B and C), with PET at top, PET/CT in middle, and CT at bottom. Maximum-intensity projection and axial images in B demonstrate multiple retroperitoneal and supradiaphragmatic lymph node metastases with intermediate PSMA expression (score 2, solid arrows). In total, 3 bone lesions (dotted arrows) define oligometastatic bone involvement. (C) Sclerotic bone metastasis in thoracic spine demonstrates low PSMA expression (score 1). Final diagnosis was miTONOM1a(RP, SD) b(oligo). 
TNM proposal and other image classification systems-initial definitions are arbitrary and not supported by strong clinical evidence. We admit that although our approach parallels the now extensively validated clinicopathologic TNM, miTNM is based only on our joint experience and the supporting evidence, with no prognostic validation having been performed. The historical development of classification systems for imaging (e.g., BIRADS, PI-RADS, RECIST, and PERCIST) demonstrates that after an initial proposal with often a limited scientific basis, further sequential adjustments have been made to optimize applicability and clinical validity. We expect and desire a similar process for the miTNM system presented here. The system will evolve as more evidence becomes available for PSMA-ligand PET/CT and patient outcome. miTNM remains inclusive for other staging systems focusing on local staging or management decisions.

Currently, there are several different PSMA ligands in clinical use. Because comparative data on biodistribution and uptake in tumors are not currently available, caution is warranted when comparisons are made between studies applying different PSMA ligands. Therefore, we highly recommend that the specific PSMA ligand be disclosed and that the same ligand be used when followup imaging is performed. Notably, this proposal focuses on small ligands, because antibodies (e.g., J591), minibodies, and other, larger, molecules with affinity to PSMA demonstrate a substantially different biodistribution and currently lack data describing their clinical use (58).

\section{FUTURE DEVELOPMENT}

PSMA-ligand PET enables unprecedented delineation of wholebody tumor burden based on high target-to-background expression levels (Supplemental Fig. 1) (59). Introduction of tools for whole-

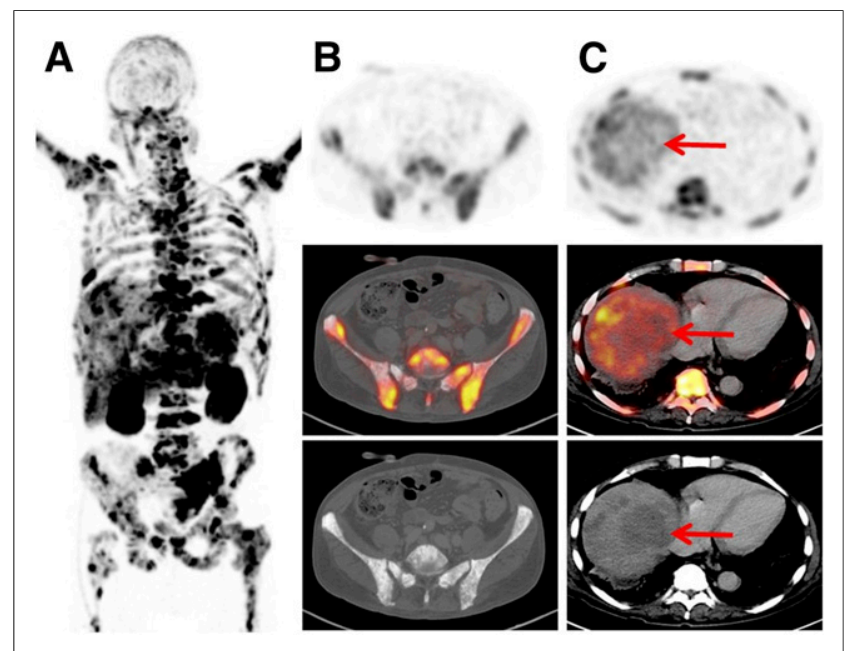

FIGURE 7. ${ }^{68} \mathrm{Ga}-\mathrm{PSMA} 11 \mathrm{PET} / \mathrm{CT}$ restaging in 76-y-old patient with advanced metastatic castration-resistant prostate cancer before potential PSMA-radioligand therapy: PET maximum-intensity projection (A) and 2 levels of axial PET/CT (B and C). PET/CT shows diffuse skeletal uptake with low to high PSMA expression (score 1-3). PET/CT shows multiple liver metastases with low PSMA expression (arrows, score 1). Final diagnosis was miTONOM1b(dmi)c(liver). PSMA-radioligand therapy was omitted because of diffuse marrow involvement and low PSMA expression in liver metastases. body tumor volumetry based on a combination of molecular and morphologic techniques might overcome several limitations of solely morphology-based criteria, such as RECIST (60): lesions without distinct morphologic boundaries, such as bone metastases, could be included in the evaluation. Molecular imaging also offers the potential to acknowledge target expression as part of a quantitative imaging biomarker, and lesions can be subselected by certain target definitions, minimizing potential bias. Consequently, direct assessment of tumor volume, instead of assessment of lesion diameter sums, could be done. For PSMA-ligand PET, initial attempts have been made by introducing PSMAderived tumor volume, total lesion PSMA, or bone PET indices $(59,61)$. Further advances in the field of software-assisted tumor delineation will help to automatically delineate-separately for bone and soft tissue-total tumor volume, total tumor target expression, or a combination of these. Prospective clinical evaluation is mandatory to assess their potential for predicting prognosis and response in patients with PSMA-expressing prostate cancer.

\section{SUMMARY}

We propose miTNM, version 1.0, as a standardized framework for reporting the results of PSMA-ligand PET/CT or PET/MRI. miTNM organizes the staging of whole-body prostate cancer by including information on exact location, pattern of disease distribution, PSMA expression, and level of certainty. miTNM aims to aid information exchange by unifying clinical and research reporting of PSMA-ligand imaging. Prospective evaluation of miTNM needs to be performed and its impact on patient prognosis and management assessed.

\section{DISCLOSURE}

Wolfgang Fendler received a scholarship from the Deutsche Forschungsgemeinschaft (grant 807122). Jeremie Calais received a grant from the Fondation ARC pour la recherche sur le cancer (grant SAE20160604150). Matthias Eiber received funding from the Deutsche Forschungsgemeinschaft within Sonderforschungsbereich 824 (project B11). No other potential conflict of interest relevant to this article was reported.

\section{ACKNOWLEDGMENTS}

We thank Marie Bieth for contributing a case demonstrating software-algorithm-based automatic delineation of tumor volume. We thank Torsten Kuwert, Christian Schmidkonz, Thorsten Derlin, Steve Rowe, and Michael A. Gorin for contributing images outlining the biodistribution of ${ }^{99 \mathrm{~m}} \mathrm{Tc}-\mathrm{MIP} 1404,{ }^{68} \mathrm{Ga}$-PSMA I\&T, and ${ }^{18}$ F-DCFPyL.

\section{REFERENCES}

1. Perera M, Papa N, Christidis D, et al. Sensitivity, specificity, and predictors of positive ${ }^{68} \mathrm{Ga}$-prostate-specific membrane antigen positron emission tomography in advanced prostate cancer: a systematic review and meta-analysis. Eur Urol. 2016;70:926-937.

2. Eiber M, Maurer T, Souvatzoglou M, et al. Evaluation of hybrid ${ }^{68} \mathrm{Ga}-\mathrm{PSMA}$ ligand PET/CT in 248 patients with biochemical recurrence after radical prostatectomy. J Nucl Med. 2015;56:668-674.

3. Eiber M, Nekolla SG, Maurer T, Weirich G, Wester HJ, Schwaiger M. ${ }^{68} \mathrm{Ga}-$ PSMA PET/MR with multimodality image analysis for primary prostate cancer. Abdom Imaging. 2015;40:1769-1771. 
4. Rauscher I, Maurer T, Beer AJ, et al. Value of ${ }^{68} \mathrm{Ga}$-PSMA HBED-CC PET for the assessment of lymph node metastases in prostate cancer patients with biochemical recurrence: comparison with histopathology after salvage lymphadenectomy. J Nucl Med. 2016;57:1713-1719.

5. Maurer T, Gschwend JE, Rauscher I, et al. Diagnostic efficacy of ${ }^{68}$ galliumPSMA positron emission tomography compared to conventional imaging for lymph node staging of 130 consecutive patients with intermediate to high risk prostate cancer. J Urol. 2016;195:1436-1443.

6. Fendler WP, Schmidt DF, Wenter V, et al. ${ }^{68} \mathrm{Ga}$-PSMA PET/CT detects the location and extent of primary prostate cancer. J Nucl Med. 2016;57:17201725 .

7. Herlemann A, Wenter V, Kretschmer A, et al. ${ }^{68} \mathrm{Ga}$-PSMA positron emission tomography/computed tomography provides accurate staging of lymph node regions prior to lymph node dissection in patients with prostate cancer. Eur Urol. 2016;70:553-557.

8. Afshar-Oromieh A, Avtzi E, Giesel FL, et al. The diagnostic value of PET/ $\mathrm{CT}$ imaging with the ${ }^{68} \mathrm{Ga}$-labelled PSMA ligand HBED-CC in the diagnosis of recurrent prostate cancer. Eur J Nucl Med Mol Imaging. 2015;42:197209.

9. Afshar-Oromieh A, Holland-Letz T, Giesel FL, et al. Diagnostic performance of ${ }^{68} \mathrm{Ga}-\mathrm{PSMA}-11$ (HBED-CC) PET/CT in patients with recurrent prostate cancer: evaluation in 1007 patients. Eur J Nucl Med Mol Imaging. 2017;44:1258-1268.

10. Afshar-Oromieh A, Malcher A, Eder M, et al. PET imaging with a $\left[{ }^{68} \mathrm{Ga}\right]-$ gallium-labelled PSMA ligand for the diagnosis of prostate cancer: biodistribution in humans and first evaluation of tumour lesions. Eur J Nucl Med Mol Imaging. 2013;40:486-495.

11. Kesch C, Vinsensia M, Radtke JP, et al. Intraindividual comparison of ${ }^{18} \mathrm{~F}-$ PSMA-1007-PET/CT, multiparametric MRI, and radical prostatectomy specimens in patients with primary prostate cancer: a retrospective, proof-of-concept study. J Nucl Med. 2017;58:1805-1810.

12. Giesel FL, Fiedler H, Stefanova M, et al. PSMA PET/CT with Glu-urea-Lys(Ahx)-[ $\left[{ }^{68} \mathrm{Ga}\right.$ (HBED-CC)] versus 3D CT volumetric lymph node assessment in recurrent prostate cancer. Eur J Nucl Med Mol Imaging. 2015;42:17941800 .

13. Pyka T, Okamoto S, Dahlbender M, et al. Comparison of bone scintigraphy and ${ }^{68} \mathrm{Ga}$-PSMA PET for skeletal staging in prostate cancer. Eur J Nucl Med Mol Imaging. 2016;43:2114-2121.

14. Afshar-Oromieh A, Zechmann CM, Malcher A, et al. Comparison of PET imaging with a ${ }^{68} \mathrm{Ga}$-labelled PSMA ligand and ${ }^{18} \mathrm{~F}$-choline-based PET/CT for the diagnosis of recurrent prostate cancer. Eur J Nucl Med Mol Imaging. 2014;41:11-20.

15. Rowe SP, Macura KJ, Mena E, et al. PSMA-based $\left[{ }^{18} \mathrm{~F}\right] \mathrm{DCFPyL}$ PET/CT is superior to conventional imaging for lesion detection in patients with metastatic prostate cancer. Mol Imaging Biol. 2016;18:411-419.

16. European Association of Urology guideline on prostate cancer. European Association of Urology website. http://uroweb.org/guideline/prostate-cancer. Accessed Jan 29, 2018.

17. Barentsz JO, Weinreb JC, Verma S, et al. Synopsis of the PI-RADS v2 guidelines for multiparametric prostate magnetic resonance imaging and recommendations for use. Eur Urol. 2016;69:41-49.

18. Imbriaco M, Larson SM, Yeung HW, et al. A new parameter for measuring metastatic bone involvement by prostate cancer: the bone scan index. Clin Cancer Res. 1998;4:1765-1772.

19. Sedgwick EL, Ebuoma L, Hamame A, et al. BI-RADS update for breast cancer caregivers. Breast Cancer Res Treat. 2015;150:243-254.

20. Tessler FN, Middleton WD, Grant EG, et al. ACR Thyroid Imaging, Reporting and Data System (TI-RADS): white paper of the ACR TI-RADS committee. J Am Coll Radiol. 2017;14:587-595.

21. Cheng L, Montironi R, Bostwick DG, Lopez-Beltran A, Berney DM. Staging of prostate cancer. Histopathology. 2012;60:87-117.

22. Hövels AM, Heesakkers RA, Adang EM, et al. The diagnostic accuracy of CT and MRI in the staging of pelvic lymph nodes in patients with prostate cancer: a meta-analysis. Clin Radiol. 2008;63:387-395.

23. Heidenreich A, Bastian PJ, Bellmunt J, et al. EAU guidelines on prostate cancer. part 1: screening, diagnosis, and local treatment with curative intent-update 2013. Eur Urol. 2014;65:124-137.

24. Jilg CA, Drendel V, Rischke HC, et al. Diagnostic accuracy of Ga-68-HBEDCC-PSMA-ligand-PET/CT before salvage lymph node dissection for recurrent prostate cancer. Theranostics. 2017;7:1770-1780.

25. Fendler WP, Calais J, Allen-Auerbach M, et al. ${ }^{68}$ Ga-PSMA-11 PET/CT interobserver agreement for prostate cancer assessments: an international multicenter prospective study. J Nucl Med. 2017;58:1617-1623.
26. Rauscher I, Maurer T, Fendler WP, Sommer WH, Schwaiger M, Eiber M. ${ }^{68} \mathrm{Ga}-$ PSMA ligand PET/CT in patients with prostate cancer: how we review and report. Cancer Imaging. 2016;16:14.

27. Fendler WP, Eiber M, Beheshti M, et al. ${ }^{68} \mathrm{Ga}$-PSMA PET/CT: joint EANM and SNMMI procedure guideline for prostate cancer imaging: version 1.0. Eur J Nucl Med Mol Imaging. 2017;44:1014-1024.

28. Fanti S, Minozzi S, Morigi JJ, et al. Development of standardized image interpretation for ${ }^{68} \mathrm{Ga}$-PSMA PET/CT to detect prostate cancer recurrent lesions. Eur J Nucl Med Mol Imaging. 2017;44:1622-1635.

29. Wright GL Jr, Grob BM, Haley C, et al. Upregulation of prostate-specific membrane antigen after androgen-deprivation therapy. Urology. 1996;48:326-334.

30. Wright GL Jr, Haley C, Beckett ML, Schellhammer PF. Expression of prostatespecific membrane antigen in normal, benign, and malignant prostate tissues. Urol Oncol. 1995;1:18-28.

31. Ross JS, Sheehan CE, Fisher HA, et al. Correlation of primary tumor prostatespecific membrane antigen expression with disease recurrence in prostate cancer. Clin Cancer Res. 2003;9:6357-6362.

32. Parimi V, Goyal R, Poropatich K, Yang XJ. Neuroendocrine differentiation of prostate cancer: a review. Am J Clin Exp Urol. 2014;2:273-285.

33. Yuan TC, Veeramani S, Lin MF. Neuroendocrine-like prostate cancer cells: neuroendocrine transdifferentiation of prostate adenocarcinoma cells. Endocr Relat Cancer. 2007;14:531-547.

34. Koerber SA, Utzinger MT, Kratochwil C, et al. ${ }^{68}$ Ga-PSMA-11 PET/CT in newly diagnosed carcinoma of the prostate: correlation of intraprostatic PSMA uptake with several clinical parameters. J Nucl Med. 2017;58:19431948.

35. Budäus L, Leyh-Bannurah SR, Salomon G, et al. Initial experience of ${ }^{68} \mathrm{Ga}-$ PSMA PET/CT imaging in high-risk prostate cancer patients prior to radical prostatectomy. Eur Urol. 2015;69:393-396.

36. Giesel FL, Hadaschik B, Cardinale J, et al. F-18 labelled PSMA-1007: biodistribution, radiation dosimetry and histopathological validation of tumor lesions in prostate cancer patients. Eur J Nucl Med Mol Imaging. 2017;44: 678-688.

37. Weinreb JC, Blume JD, Coakley FV, et al. Prostate cancer: sextant localization at MR imaging and MR spectroscopic imaging before prostatectomy-results of ACRIN prospective multi-institutional clinicopathologic study. Radiology. 2009; 251:122-133.

38. Zettinig O, Shah A, Hennersperger C, et al. Multimodal image-guided prostate fusion biopsy based on automatic deformable registration. Int J Comput Assist Radiol Surg. 2015;10:1997-2007.

39. Costa DN, Pedrosa I, Donato F Jr, Roehrborn CG, Rofsky NMMR. Imagingtransrectal US fusion for targeted prostate biopsies: implications for diagnosis and clinical management. Radiographics. 2015;35:696-708.

40. Moore CM, Kasivisvanathan V, Eggener S, et al. Standards of reporting for MRItargeted biopsy studies (START) of the prostate: recommendations from an International Working Group. Eur Urol. 2013;64:544-552.

41. Rosenkrantz AB, Verma S, Choyke P, et al. Prostate magnetic resonance imaging and magnetic resonance imaging targeted biopsy in patients with a prior negative biopsy: a consensus statement by AUA and SAR. J Urol. 2016;196:1613-1618.

42. Eiber M, Weirich G, Holzapfel K, et al. Simultaneous ${ }^{68}$ Ga-PSMA HBED-CC PET/MRI improves the localization of primary prostate cancer. Eur Urol. 2016; 70:829-836.

43. Salomon L, Colombel M, Patard JJ, Gasman D, Chopin D, Abbou CC. Prostate biopsy in the staging of prostate cancer. Prostate Cancer Prostatic Dis. 1997;1:54-58.

44. Vallancien G, Bochereau G, Wetzel O, Bretheau D, Prapotnich D, Bougaran J. Influence of preoperative positive seminal vesicle biopsy on the staging of prostatic cancer. J Urol. 1994;152:1152-1156.

45. Daneshmand S, Quek ML, Stein JP, et al. Prognosis of patients with lymph node positive prostate cancer following radical prostatectomy: long-term results. J Urol. 2004;172:2252-2255.

46. Boorjian SA, Thompson RH, Siddiqui S, et al. Long-term outcome after radical prostatectomy for patients with lymph node positive prostate cancer in the prostate specific antigen era. J Urol. 2007;178:864-870.

47. Budiharto T, Joniau S, Lerut E, et al. Prospective evaluation of ${ }^{11} \mathrm{C}$-choline positron emission tomography/computed tomography and diffusion-weighted magnetic resonance imaging for the nodal staging of prostate cancer with a high risk of lymph node metastases. Eur Urol. 2011;60:125-130.

48. Joniau S, Van den Bergh L, Lerut E, et al. Mapping of pelvic lymph node metastases in prostate cancer. Eur Urol. 2013;63:450-458.

49. Paño B, Sebastià C, Buñesch L, et al. Pathways of lymphatic spread in male urogenital pelvic malignancies. Radiographics. 2011;31:135-160. 
50. Krohn T, Verburg FA, Pufe T, et al. $\left[{ }^{68} \mathrm{Ga}\right.$ PSMA-HBED uptake mimicking lymph node metastasis in coeliac ganglia: an important pitfall in clinical practice. Eur J Nucl Med Mol Imaging. 2015;42:210-214.

51. Sheikhbahaei S, Afshar-Oromieh A, Eiber M, et al. Pearls and pitfalls in clinical interpretation of prostate-specific membrane antigen (PSMA)-targeted PET imaging. Eur J Nucl Med Mol Imaging. 2017;44:2117-2136.

52. Schweizer MT, Zhou XC, Wang H, et al. Metastasis-free survival is associated with overall survival in men with PSA-recurrent prostate cancer treated with deferred androgen deprivation therapy. Ann Oncol. 2013;24:28812886.

53. Tosoian JJ, Gorin MA, Ross AE, Pienta KJ, Tran PT, Schaeffer EM. Oligometastatic prostate cancer: definitions, clinical outcomes, and treatment considerations. Nat Rev Urol. 2017;14:15-25.

54. Ost P, Decaestecker K, Lambert B, et al. Prognostic factors influencing prostate cancer-specific survival in non-castrate patients with metastatic prostate cancer. Prostate. 2014;74:297-305.

55. Parker C, Nilsson S, Heinrich D, et al. Alpha emitter radium-223 and survival in metastatic prostate cancer. N Engl J Med. 2013;369:213-223.
56. Rahbar K, Ahmadzadehfar H, Kratochwil C, et al. German multicenter study investigating ${ }^{177} \mathrm{Lu}-\mathrm{PSMA}-617$ radioligand therapy in advanced prostate cancer patients. J Nucl Med. 2017;58:85-90.

57. Jong JM, Oprea-Lager DE, Hooft L, et al. Radiopharmaceuticals for palliation of bone pain in patients with castration-resistant prostate cancer metastatic to bone: a systematic review. Eur Urol. 2016;70:416-426.

58. Pandit-Taskar N, O'Donoghue JA, Durack JC, et al. A phase I/II study for analytic validation of ${ }^{89} \mathrm{Zr}-\mathrm{J} 591$ immunoPET as a molecular imaging agent for metastatic prostate cancer. Clin Cancer Res. 2015;21:5277-5285.

59. Bieth M, Krönke M, Tauber R, et al. Exploring new multimodal quantitative imaging indices for the assessment of osseous tumour burden in prostate cancer using ${ }^{68}$ Ga-PSMA-PET/CT. J Nucl Med. 2017;58:1632-1637.

60. Eisenhauer EA, Therasse P, Bogaerts J, et al. New response evaluation criteria in solid tumours: revised RECIST guideline (version 1.1). Eur J Cancer. 2009;45:228-247.

61. Schmuck S, von Klot CA, Henkenberens C, et al. Initial experience with volumetric ${ }^{68} \mathrm{Ga}$-PSMA I\&T PET/CT for assessment of whole-body tumor burden as a quantitative imaging biomarker in patients with prostate cancer. $J$ Nucl Med. 2017;58:1962-1968. 